\title{
How tribology has been helping us to advance and to survive
}

\author{
Gwidon W. STACHOWIAK* \\ Tribology Laboratory, School of Mechanical and Civil Engineering Curtin University, Bentley, Western Australia 6102, Australia \\ Received: 09 May 2017 / Revised: 25 May 2017 / Accepted: 07 June 2017 \\ (C) The author(s) 2017. This article is published with open access at Springerlink.com
}

\begin{abstract}
Movement between contacting surfaces ranges from macro to micro scales, from the movement of continental plates and glaciers to the locomotion of animals and insects. Surface topographies, lubricant layers, contaminants, operating conditions, and others control it, i.e., this movement depends on the tribological characteristics of a system. Before the industrial revolution, friction and wear were controlled by the application of animal fat or oil. During the industrial revolution, with the introduction of trains and other machinery, the operating conditions at the contacting surfaces changed dramatically. New bearings were designed and built and simple lubrication measures were no longer satisfactory. It became critical to understand the lubrication mechanisms involved. During that period, solid theoretical foundations, leading to the development of new technologies, were laid. The field of tribology had gained a significant prominence, i.e., it became clear that without advancements in tribology the technological progress would be limited. It was no longer necessary to build oversized ship bearings hoping that they would work. The ship or automobile bearings could now be optimized and their behavior predicted. By the middle of the 20th century, lubrication mechanisms in nonconformal contacts, i.e., in gears, rolling contact bearings, cams and tappets, etc., were also finally understood.

Today, we face new challenges such as sustainability, climate change and gradual degradation of the environment. Problems of providing enough food, clean water and sufficient energy to the human population to pursue a civilized life still remain largely unsolved. These challenges require new solutions and innovative approaches. As the humanity progresses, tribology continue to make vital contributions in addressing the demands for advanced technological developments, resulting in, for example, reducing the fuel consumption and greenhouse gases emission, increasing machine durability and improving the quality of life through artificial implants, among the others.
\end{abstract}

Keywords: tribology; friction; lubrication and wear

\section{Tribology as part of our lives}

In our everyday life we take many things for granted. It never occurs to us to pause and think why our hands or feet provide a perfect grip on most of the surfaces. We rarely think why sharks swim so fast or why geckos can walk on glass surface even when upside down. We expect spacecraft or satellites that we send to explore our solar system and beyond to operate smoothly even after many months or years of being idle. We expect that our trains and aircraft would stop exactly at the designated places at train stations and airports. When hopping into a car we don't think twice about the material used for the car seats. We don't think often why the tectonic plates or glaciers move with apparent ease. These seemingly diverse problems, and many others, are of great interest and research focus of tribologists. Tribology has helped us to discover not only the underlying mechanisms involved but also how to utilize these findings in practical applications.

We now understand why, as the response to stress,

* Corresponding author: Gwidon W. STACHOWIAK, E-mail: Gwidon.Stachowiak@curtin.edu.au 
our hands and feet sweat. The reason is to provide a perfect grip to either hold a weapon firmly or to facilitate a rapid escape. We found that a special skin texture combined with streamlined bodies allows sharks to swim very fast. We managed to duplicate this texture on artificial surfaces with potential applications ranging from swimsuits to submarines. The gecko's ability to climb vertical walls and walk on ceilings lies in the structure of their feet containing complex hierarchical arrangements of lamellae, setae, branches and spatula. These billions of spatula bond to the surface by long range van der Waals forces [1-4]. However, it seems that the electrostatic effects cannot completely be ruled out as they might be contributing to the gecko's adhesion on some surfaces [4]. Attempts to duplicate these remarkable features of the gecko's feet in manufacturing self-cleaning, re-attachable dry adhesive tapes have already been made [5].

As the humanity progressed, new technologies, devices, materials and surface treatments required novel lubricants and lubrication systems. The technological advancements, like the development of high-speed trains, aircraft, space stations, computer hard discs, artificial implants, and many other engineering and bio-medical systems, have only been possible through the advances in tribology. For example, the question of how to safely stop a 16 car (about $400 \mathrm{~m}$ long) high-speed train travelling at $280 \mathrm{~km} / \mathrm{h}$ or more, or A380 travelling at $250 \mathrm{~km} / \mathrm{h}$ on landing and weighing almost 400 tones is an important one. When brakes are applied a large proportion of the kinetic energy of the train or the plane is dissipated as heat. Traditionally used brake material would crack due to the thermal stress. Therefore carbon fiber brakes are used instead.

A new technological frontier of space also demanded urgent tribological solutions. In space the environment is extreme: temperatures are below $-200{ }^{\circ} \mathrm{C}$, there is vacuum and radiation. The temperature gradients are very large since the metal surface can heat up to $+250{ }^{\circ} \mathrm{C}$ when exposed to the sun. Traditionally used lubricants wouldn't work, as they would either freeze, evaporate or decompose under radiation. As there is no oxygen and water in space no friction reducing oxide layers could grow to provide some form of a lubricating solid film on the surfaces. To reduce friction and combat wear in space new surface coatings suitable for vacuum conditions have therefore been developed. These coatings consist of a thin layer of soft film, typically molybdenum disulphide, artificially deposited on the surfaces. Coatings of solid lubricant are built up atom by atom yielding a mechanically strong surface layer with a long life service and the minimum quantity of solid lubricant [6].

There is also science behind the material selection for car seats, as the material chosen must perform well with different fabrics/leathers that we wear. Neither low nor high friction materials for car seats would be popular with the users.

At the macroscale, the layers of water and fragmented rock dictate the movement of glaciers or geological plates, i.e., this movement is controlled by the tribological principles.

Friction and wear are accepted as an integral part of our lives and we often take their effects for granted. For example, we notice how crucial the effect of friction is on our walk especially when the friction dramatically drops, i.e., when the surface is slippery and we fall. When there is a problem with friction or wear then we seek a technological solution. For example, if the roads were slippery all the time, like after the first autumn rain, then we would quickly develop a technology to provide safe driving and braking under those conditions.

There is an inevitable cost related to wear and friction. Wear results in continuous renewal of our possessions and costs energy. Industries producing shoes, car tyres, slurry pumps, etc., would suffer enormously if their products did not wear. The price we pay for this is high as energy and materials are consumed to replace the worn items. Mining minerals, crude oil and gas requires energy. Further energy is needed to transform the ore into metals, crude oil into petroleum, etc. But this is not the end of it. Energy is needed to manufacture and transport the components produced across the globe and also to overcome friction in machine elements such as pistons, gears and bearings. The friction between moving machine elements in particular results in waste of an enormous amount of energy. Rapidly growing human population with a strong appetite for new products, combined 
with our limited energy resources, poses a serious challenge to the researchers in tribology. Our resources and energy are in finite supply and hence the need to control wear and friction becomes increasingly urgent. In addition, the rapid spread of the lifestyle from the advanced countries to the developing countries inevitably brings extra demands and pressures on the available resources and energy. The study of friction and wear would therefore continue to provide many challenges for the researchers in tribology for many decades to come.

\section{Lubrication to combat friction}

Long before the initiation of any historical records, the early humans used basic tribological principles in their everyday lives. For example, the importance of friction and sliding speed was quickly recognized when rubbing two sticks together to make fire. To rotate the stick faster a bow was utilized. How the humans discovered this principle of the temperature rise in sliding contact that was then used to light the fire is unknown. Perhaps prehistoric people had noticed that hands warm up when rubbed together and then tried this with the sticks. We'll never know. The ancient history contains abundant examples of the applied study of friction and wear. For example, lubricants were used in sledges to move large stone blocks for the construction of the pyramids at Giza or to move massive monuments; a wheel with a lubricated bearing was developed for chariots and carts, etc. [7].

The effect of fat on friction reduction was probably known long before the recorded history. At the beginning, the prehistoric farmers experimented with animal fat to lubricate the axles of their carts before embarking on a more ambitious task of manufacturing first grease. The animal fat was mixed with soda. The mixture, when placed in the cart's bearing, turned into grease with the help of frictional heat. At that time the concept of a lubricant and lubrication was born. People found that to prevent the axles from overheating was to keep them lubricated. This is a basic principle behind reducing friction and wear, i.e., to make sure that a layer of lubricant is present between the sliding surfaces all the time. This phenomenon was easily observed but to put science behind it was a far more difficult task, and centuries had elapsed before solid theoretical foundations of lubrication were laid.

The pioneering study of a lubrication mechanism between two conformal sliding surfaces was conducted towards the end of the 19th century. As usually is the case, necessity dictated the rigorous scientific research into the mechanisms behind the lubrication process. During the industrial revolution railways were developed and used on regular basis in England. However, the railway axle bearings were a continuous source of problems. As rolling contact bearings were not yet commercially available, these were simple journal bearings with lubricating holes located on the top. The problem was that often, during the operation, these bearings ceased to rotate or became very hot due to excessive friction, frequently catching a fire. Stopping the wheel rotation resulted in a flat spot on its rolling surface, rendering the wheel practically useless. Wheels were costly to replace and repair.

Thus in 1896 the Institution of Mechanical Engineers commissioned one of its top engineers, Beauchamp Tower, to investigate this problem. For the first time a systematic and detailed study of the friction in journal bearings was performed. However, the problem was so unusual that after a few months of testing the issue of high friction in these bearings was still largely unresolved. As often happens in science a chance intervened with a lucky discovery. When bearings were running in the laboratory it was noticed that the lubricating holes were persistently leaking oil. Plugging leaking holes with rags and then with wooden bungs didn't help much. Tower then realized that the oil in the bearing must be under a considerable pressure. When the pressure was measured it became clear that it was high enough to support the bearing load [8] (Fig. 1). What's more, the high pressure implied that between the bearing and shaft surfaces there was a layer of a lubricant of sufficient thickness to separate them. The solution was to remove the lubrication hole and instead fit a container with oil underneath. This was a major discovery and at that point the existing knowledge of lubrication and its effects on friction and wear was transformed forever.

Tower's discovery provided strong experimental evidence supporting the hydrodynamic theory of lubrication that was being developed at the time by 

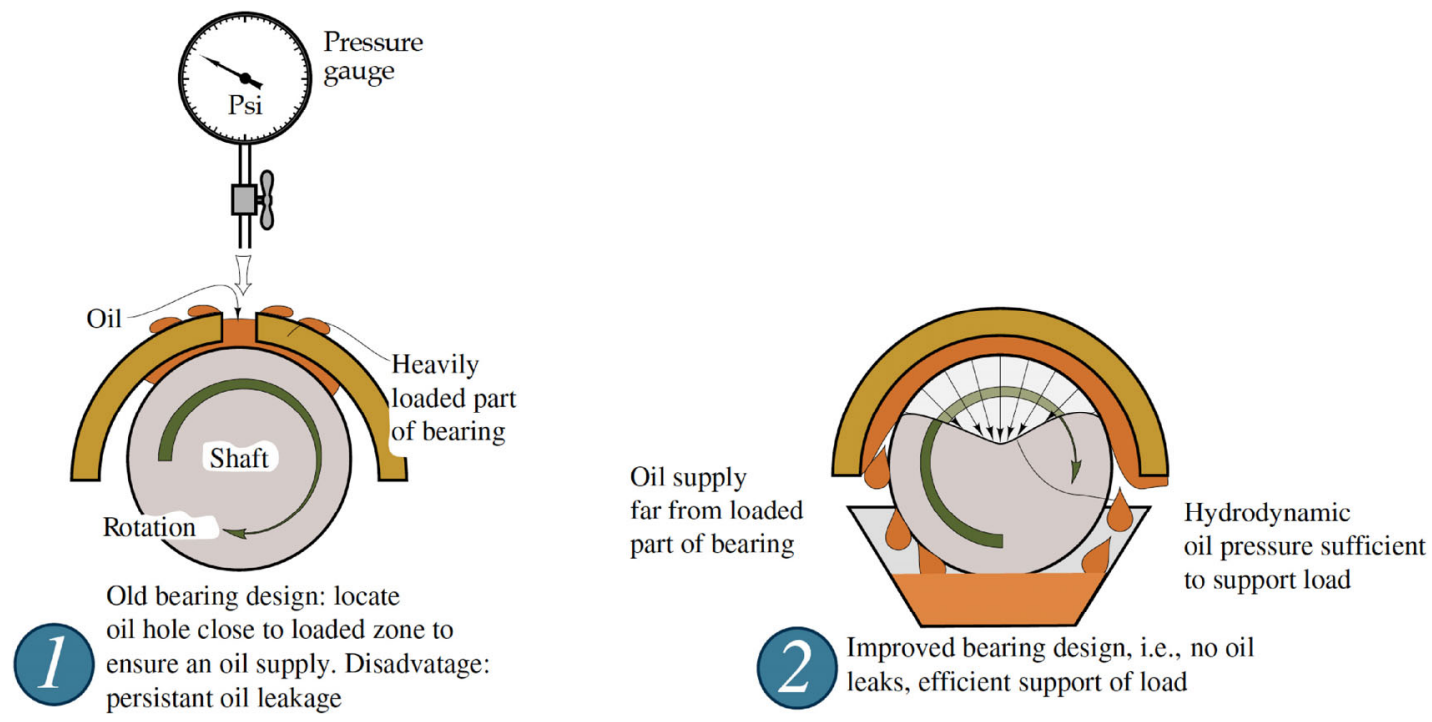

Fig. 1 Discovery of hydrodynamic lubrication in axle bearing (adapted from Ref. [9]).

Reynolds [10]. It needs to be mentioned that the concept of a film of lubricant separating two sliding surfaces was not new. It had been proposed earlier by Leupold in 1735, Leslie in 1804, Rennie in 1829, Adams in 1853 and Hirn in 1854 [7]. What was new, however, was the science, encapsulated in a set of elegant mathematical equations, neatly describing the lubrication mechanism in action. A combination of Tower's experimental results and Reynolds' mathematical analysis provided a vital tool in bearing design. It replaced numerous empirical ideas on railway axle bearing lubrication and effectively solved the problem. In this early lubrication research, Tower's measurements did not include other fundamental bearing parameters, such as operating temperature, elastic deformation of the bearing under load or inertia effects. This was done much later [11].

By the middle of the 20th century the lubrication mechanisms in conformal contacts in hydrodynamic and hydrostatic bearings had been well studied, understood and defined. However, the mechanism of lubrication operating in highly loaded non-conformal contacts remained a mystery for some time. Nonconformal contacts are commonly found in gears, rolling contact bearings, cams and tappets, etc., and the contact areas and stresses are very much different from those encountered in conformal, hydrodynamic contacts. The contact areas are very small and the resulting contact pressures are much higher, over
1 GPa. In 1880 Heinrich Hertz developed neat stress formulas for various non-conformal contact geometries [12], which are still in use today.

It was known that a very thin lubricating film existed between gear teeth. The thickness of these films, once measured, was far too low to be reliably predicted by the classical hydrodynamic theory [13]. Two Russian researchers, Ertel and Grubin, solved the mystery and provided the answer. They realized that a combination of three effects: hydrodynamics, elastic deformation of the metal surfaces and the increase in the oil's viscosity under extreme pressures found in these heavily loaded non-conformal contacts, contributed to the lubrication mechanism operating $[14,15]$. Hence the mechanism was named elastohydrodynamic lubrication (EHL), which effectively means that the contacting surfaces deform elastically under the hydrodynamic pressure generated in a thin layer of the lubricating film. These lubricating films are extremely thin, in the range of 0.1 to $1 \mu \mathrm{m}$. However, despite their low thickness these films still manage to effectively separate the interacting surfaces, resulting in a significant reduction of wear and friction.

For many years little was known about the nature of the EHL films. Initially, the thickness of these films could only be estimated using electrical resistance or capacitance methods but the accurate confirmation was lacking for some time. In the 1960s Cameron and Gohar $[16,17]$ provided the answer. They devised a 
simple test comprising a glass disc and a bearing ball. The glass disc was covered with the semi-reflective coating allowing for half the light to be reflected off the glass surface and half to pass through the oil film and be reflected off the steel ball. The elastohydrodynamic films observed were thin enough to provide a good interference effect with visible light. The observations showed that under large contact stresses the surface of the ball deformed elastically to produce a continuous oil entrapment in the shape of a "horseshoe" on the edges of the contact. This elastic oil entrapment effect, shown in Fig. 2, is commonly known as the "end constriction". The elastic deformation at the constriction is lower than that in the centre. Under high contact pressures the lubricant starts behaving as a "solid material", i.e., the steel surface deforms elastically around the lubricant, at the edges of the contact. With this technique EHL films in "soft" contacts with polymethylmethacrylate and polyurethane have also been measured [18].

The EHL problem was far too complex for the analytical analysis. The simultaneous solution of sets of equations describing the hydrodynamic effect due to the relative motion of the surfaces, elastic deformations and changes in the lubricant's rheology due to very high contact pressures was impossible to achieve analytically. Dowson and Higginson accomplished this task employing numerical methods. They used the Ferranti-Pegasus valve-based computer to figure out the solution. The computer contained
3,000 valves and it took Dowson and Higginson 3,000 hours to get enough results for their paper. Their efforts resulted in an elegant formula for the elastohydrodynamic film thickness, which is still used today [19].

The application of computers in solving lubrication problems was a major breakthrough with far reaching implications. After this our approach to hydrodynamic or EHL lubrication was never the same. Before, there was always a gap between what was required in the real engineering world and the solutions available. With this new computational approach it was now possible to incorporate in the analysis of bearings common features such as heat transfer from a bearing to its housing. But, numerical methods not only provided tools to solve differential equations and complex engineering problems but also helped to gain the general understanding of the physical phenomena occurring.

Over the last several decades, significant progress has been made in both the EHL film measurement methods and in our understanding of the phenomena occurring in the EHL contacts. Main limitations of the original interferometry technique are its resolution and the requirement that one of the contacting bodies must be transparent. As the measurement resolution is dictated by the wavelength of visible light, films of thickness lower that $0.1 \mu \mathrm{m}$ could not be accurately measured.

The application of an additional solid spacer layer

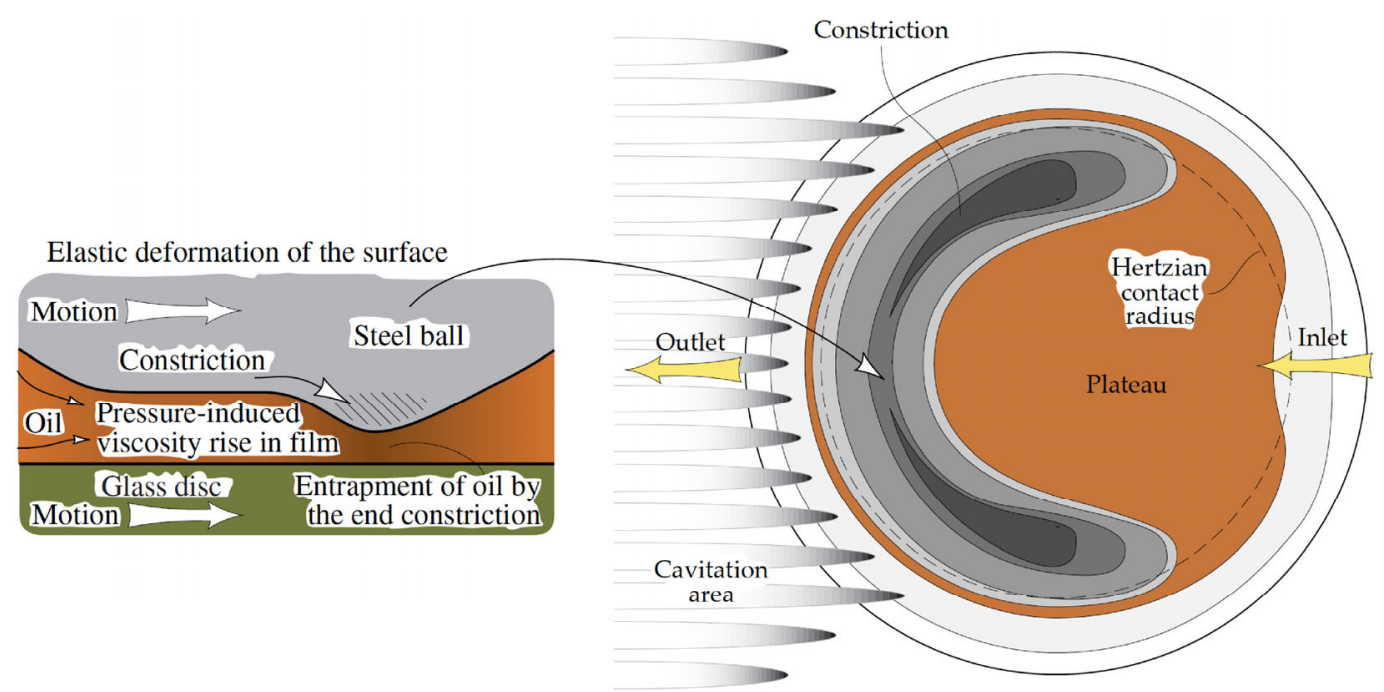

Fig. 2 Elastic oil entrapment effect in elastohydrodynamic lubrication (adapted from Refs. [9, 15]). 
combined with spectrometric analysis of the light reflected from the contact provided a significant improvement and allowed for the measurement and mapping of very thin lubricant films, down to $10 \mathrm{~nm}$ $[20,21]$. With this new technology it appeared that observations and thickness measurements of boundary films might be within the reach. However, the problem is that most of the real contacts in machine components are metal-to-metal. Thus, the transparent body requirement is a constraint for the optical interferometry and limits its use as essentially a laboratory technique. The studies of thin films and the influence of metallic surfaces on the film chemistry became possible later with the application of precise capacitance measurements using LCR meters with automatic balancing bridges [22].

A unique feature of EHL contacts is that they can provide traction. The difference between traction and friction is in the way the mechanical energy is processed. In the case of traction this energy is transmitted between the contacting bodies (i.e., one body is driving another) while with friction it is dissipated [15]. The traction is sufficiently high for the engineering applications like, for example, variable speed transmissions. The attractiveness of these transmissions lies in their ability to maintain infinitely variable output speed and almost a constant torque over the speed range. These features and low noise of these devices make them very attractive for applications in machine tools, textile industry and also in motor vehicles. The EHL traction has been studied in detail, followed by the development of traction fluids and advanced traction drives.

Recently, surface textures have been investigated, in both hydrodynamic and EHL contacts, as a means of reducing friction and wear and also improving performance. Small dimples in micrometre size are introduced into the surfaces resulting in friction and wear reduction. The topic is thoroughly investigated both experimentally and theoretically/numerically. New numerical methods simulating the dimple effects on friction and load capacity in hydrodynamic contacts are continuously being developed [23].

In many practical applications there are cases where the operating conditions are such that neither hydrodynamic nor elastohydrodynamic lubrication is effective. The question then is: how are the interacting machine components lubricated and what is the lubrication mechanism involved? The traditional name for this type of lubrication is "boundary lubrication" or more precisely "boundary and extreme-pressure lubrication". Neither of these terms describes accurately the processes at work since these concepts had been conceived long before any fundamental understanding of the mechanisms was available. Boundary and extreme-pressure lubrication is a combination of complex phenomena depending on lubricant properties, contacting body characteristics and operating contact conditions. The lubrication mechanisms involved can be classified in terms of relative load and limiting frictional temperature [15]. The lubrication mechanism is mostly controlled by the additives present in the oil. Since the cost of lubricant additives is almost negligible compared to the value of the mechanical equipment, the commercial benefits involved in this type of lubrication are large. In general, boundary and extreme-pressure lubrication involves the formation of low-friction, protective layers on the wearing surfaces. First mechanisms, i.e., the adsorption model of lubrication, were postulated by Hardy and Doubleday [24,25] and later developed by Bowden and Tabor [26] followed by many others.

The pioneering work of Bowden and Tabor has been extended by the development of a new area in tribology called tribochemistry which focuses on the chemical reactions taking place between the lubricant and surfaces under boundary lubrication conditions [27]. Numerous researchers, notably Stephen Hsu, Nic Spencer, Keiji Nakayama, Eddy Tysoe, Jean Michel Martin, Nobuo Ohmae and others, laid the solid foundations for this new branch of tribology.

\section{Wear}

From the beginning of our civilization until the $19^{\text {th }}$ century wear was routinely accepted as inevitable part of life which forces continuous renewal of most of our possessions. Items like shoes, ploughs, cart bearings and emerging machines were wearing out only to be replaced by new components. This process of continuous renewal has many benefits as it keeps the economy going, but it has also many disadvantages 
since resources and energy are consumed to replace the worn items. Friction wastes an enormous amount of energy. From the moment petroleum emerges from the ground to when it is burnt as fuel, there are frictional losses. However, more than 100 years ago, there was one wear problem which could not be easily accepted, i.e., the problem with wear of gold coins in circulation in Great Britain.

In 1898, King George III commissioned Charles Hatchett to investigate whether this weight loss could be attributed to normal, everyday wear. To investigate the problem, in a systematic way, Hatchett built a special tribometer to evaluate the wear rate of the coins. After extensive testing Hatchett concluded that rapid coins wear due to their everyday use could not account for their rapid weight loss $[7,28]$. In his experiments Hattchet demonstrated that wear can be assessed in a systematic way.

Much earlier Desaguliers hypothesized that friction and wear between clean surfaces depended on the mutual adhesion of the contacting solids [29]. This concept remained as a valid model for almost two hundred years. The experimental work of Bowden and Tabor [30] into adhesion and friction between clean metals enhanced this theory and dramatically advanced our understanding of friction and wear. Despite its initial universal acceptance, the theory linking friction to adhesive bonding between contacting surface asperities and wear has since been modified and further advanced in the light of new experimental and numerical simulations evidence conducted the atomic level [31].

In another research, about two hundred years ago in 1804, Leslie provided the first model of the friction between contaminated surfaces where waves of deformed material were pushed across the surface by the asperities from the opposing surface [32].
This theory remained obscure until 1984. Then, the experimental confirmation of material deformation and waves formation was provided by Challen, McLean and Oxley $[33,34]$. A wearing contact was modelled by a prism of hard material sliding on a block of softer material as shown in Fig. 3 . When the rigid prism was forced into the softer material and driven horizontally along the surface of the soft counterface, a wedge of deformed material accumulated in front of the prism. Undeformed material from the soft counterface flowed through this wedge, which remained at constant size. The net effect on the counterface was a layer of highly strained material. This simple model, based on slip-line fields, explained well the fundamental mechanism of abrasive wear by plastic deformation.

This study confirmed earlier observations of similar highly strained layers present on the worn surfaces [35] as well as the raised humps of material similar to the wedge $[36,37]$. So it was concluded that a large proportion of the frictional energy in unlubricated sliding is dissipated in driving waves of deformed material across the surface [38]. Since then our knowledge on wear has greatly advanced. We now understand most of the wear mechanisms, and how they are influenced by materials, lubricants, operating and environmental conditions. This understanding has been essential in the design of modern machines with required long life expectancy. However, we did not stop there as further research reveals wear mechanisms operating not only at macro scales but also at micro and nano scales.

\section{Biotribology}

Biotribology as a new area in tribology has developed as a response to the growing interest in applying
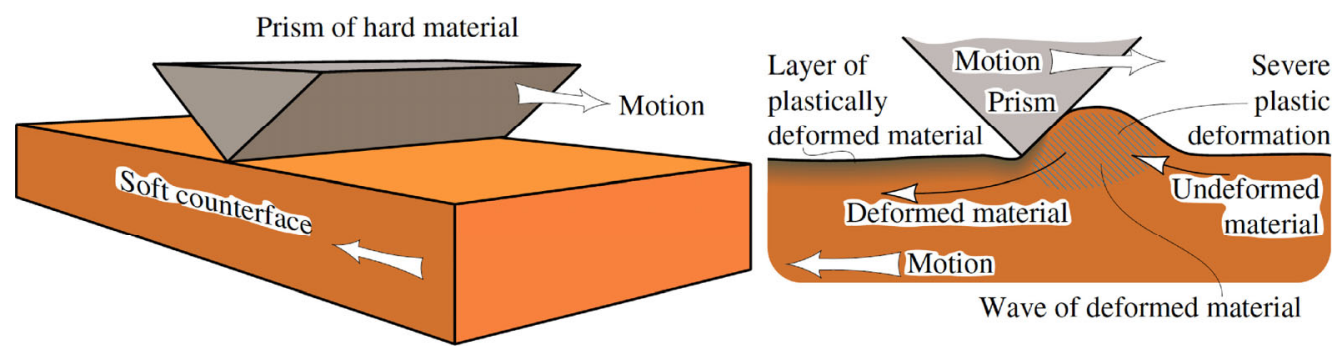

Fig. 3 Model of surface waves formation (adapted from Ref. [9]). 
scientific methods to understand tribological phenomena occurring in a human body. Animal and human bodies evolved with many sliding and frictional living tissue interfaces. One of the most important examples is the interface between the external object and the skin. Oily, friction and wear-reducing substance called "sebum" is secreted by skin to improve grip by a hand or to protect feet during walking/running. Inside the body in the lungs the bronchioles can freely dilate and contract during respiration (breathing). For this to happen, an effective lubrication facilitating this function is essential. A lubricating film on the exterior of the lungs is vital to the movement of lungs within the ribcage. Similar films are essential to the movement of eyeballs. In fact, lubricating films separate most of the internal organs, e.g., liver, intestines, etc.

The human body has more than one hundred articulating joints, which act as bearings, e.g., knees, hips, feet, spine, hands, etc. These bearings facilitate walking, running, jumping, flexing of the limbs, bending, gripping by the hands, etc. The joints consist of mostly conformal cartilaginous surfaces sliding past each other. Most synovial joints exhibit very low friction coefficients and wear. The most common problem with synovial joints is arthritis, with two principal forms: osteoarthritis and rheumatoid arthritis. Osteoarthritis, characterized by the loss of articular cartilage, meniscal tears and maceration, osteophytes, and microstructural changes in the subchondral bone, is different from rheumatoid arthritis, which occurs when the body's immune system is induced to attack the synovial joints, in particular causing damage to the articular cartilage.

Osteoarthritis is a leading cause of disability among middle-aged and elderly persons. For example, in Australia alone, osteoarthritis affects more than 50\% of the population over 65 , at a cost of over 25 billion dollars per annum to the economy and health system $[39,40]$. Despite its high cost to many societies across the world, there are no effective treatments for the disease or even symptoms relief (pain, stiffness), with the exception of weight loss and joint replacement.

Tribology has played a significant role in our understanding of osteoarthritis. Biological studies of synovial joints involve problems outside the normal range of engineering studies. However, the experimental methodologies used in studies of wear and friction of engineering materials were adapted to study wear and friction in synovial joints [41-43]. Experimental research data on wear, especially of "live" synovial joints, is notoriously difficult to obtain. Cadaver sheep joints were tested on a specially designed joint simulator in an oxygen-free sterile environment. The experiments conducted revealed that the wear particles generated in the sheep joints subjected to wear were very similar to those observed in osteoarthritic human joints. Vital conclusions about the wear mechanisms occurring in these joints were obtained. New measures were developed sensitive enough to detect minute changes occurring in knee bones and trained classifiers can now accurately predict the osteoarthritis (OA). The same principles can be used in the development of prognostic tools for, e.g., hip, hand, foot, elbow and shoulder OA and even cancers.

How is this possible? So far, "classical" surfaces have been characterized using traditional/standard surface roughness parameters, which work well with isotropic surfaces. However, many modern surfaces manufactured to suit specific performance requirements or applications often contain complex texture patterns that vary locally in roughness and directionality at different scales. There is a large variety of surface textures/patterns produced. From a viewpoint of production and application of these surfaces they need to be characterized, in the same way as the "classical" surfaces, except that different measures must be used as the standard parameters are no longer suitable. Current ASME (American Society of Mechanical Engineers) and ISO (International Organization for Standardization) surface texture standards fail to adequately describe advanced surfaces, i.e., they are either not suitable, or exhibit significant limitations. For example, how can we characterize, using traditional parameters, surfaces textures like those shown in Figs. 4 or 5? The answer might be provided by the directional fractal signature (DFS) methods, which involve the calculation of fractal dimensions in different directions and at individual scales [44]. The DFS techniques not only calculate the changes in the surface topography at different scales but also describe the surface anisotropy and show the surface dominant direction. It can be seen from 
Fig. 4 that at the largest scale of $70 \mu \mathrm{m}$ the surface changes its directionality which may affect its properties or performance, e.g., microlubrication. The DFS methods are also applicable to the characterization of surfaces which are otherwise difficult or even impossible to analyze by any other existing technique, e.g., self-structured surfaces shown in Fig. 5.

DFS methods have been adapted for use in early detection and prediction of osteoarthritis based on X-ray images of the knee or hand joints [45-51]. X-ray images of bones in knee and hand joints can be analyzed using the same DFS techniques that are used to analyze 3-D surfaces. This illustrated in Fig. 6 where the range images (with surface elevations encoded in the pixel brightness values) of a 3D surface and trabecular bone X-ray image are shown. The DFS technique can be used to accurately characterize both images.

As the osteoarthritis progresses, at some stage the joint's disease becomes untreatable by conventional means, e.g., drugs, physiotherapy, etc., and the question is: what are our options when nothing else works? The answer is in joint replacements, resurfacing and cartilage repair.

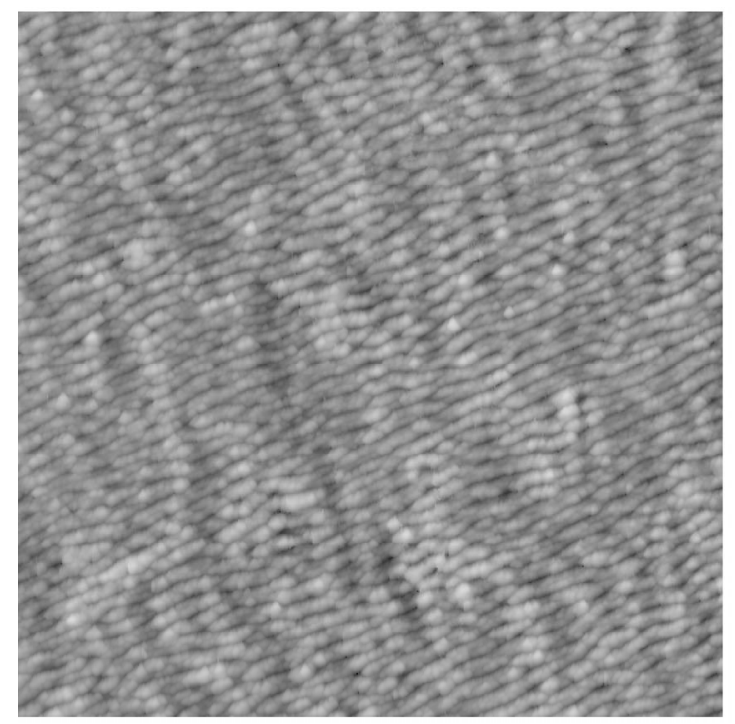

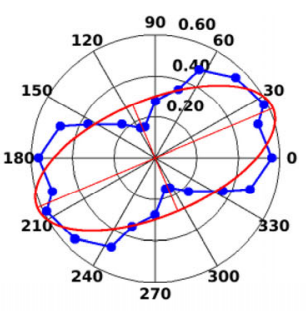

Scale: $30 \mu \mathrm{m}$

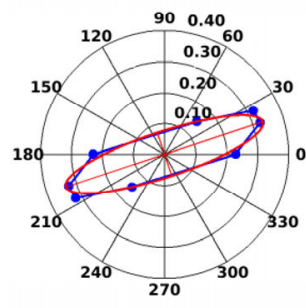

Scale: $55 \mu \mathrm{m}$

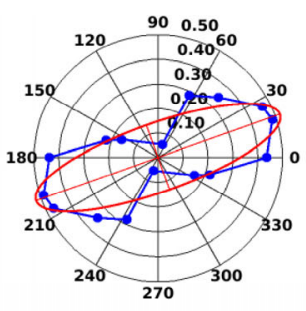

Scale: $40 \mu \mathrm{m}$

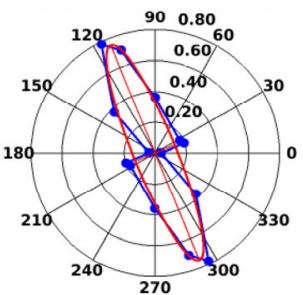

Scale: $70 \mu \mathrm{m}$

Fig. 4 Image of the textured surface together with the DFS analysis results presented as Rose plots at four scales.

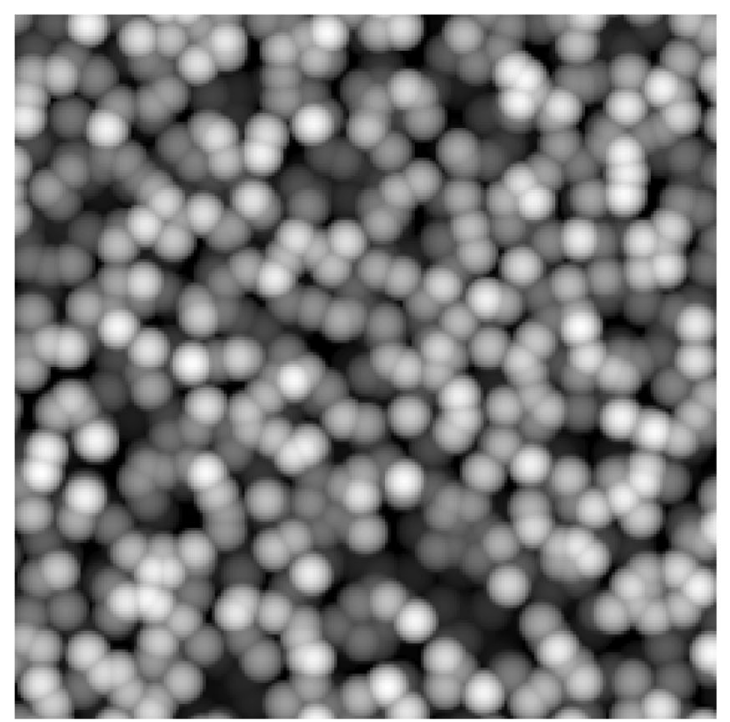

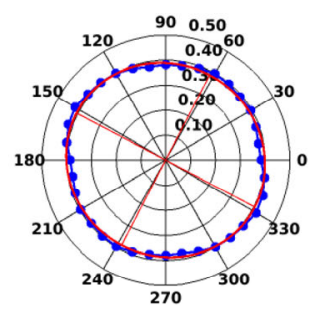

Scale: 6 pixels

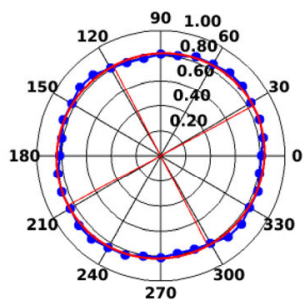

Scale: 11 pixels

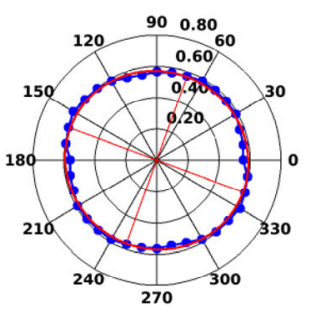

Scale: 8 pixels

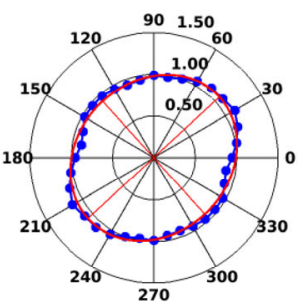

Scale: 14 pixels

Fig. 5 Image of the self-structured surface together with the DFS analysis results using augmented blanket with rotating grid method (adapted from Ref. [48]). 


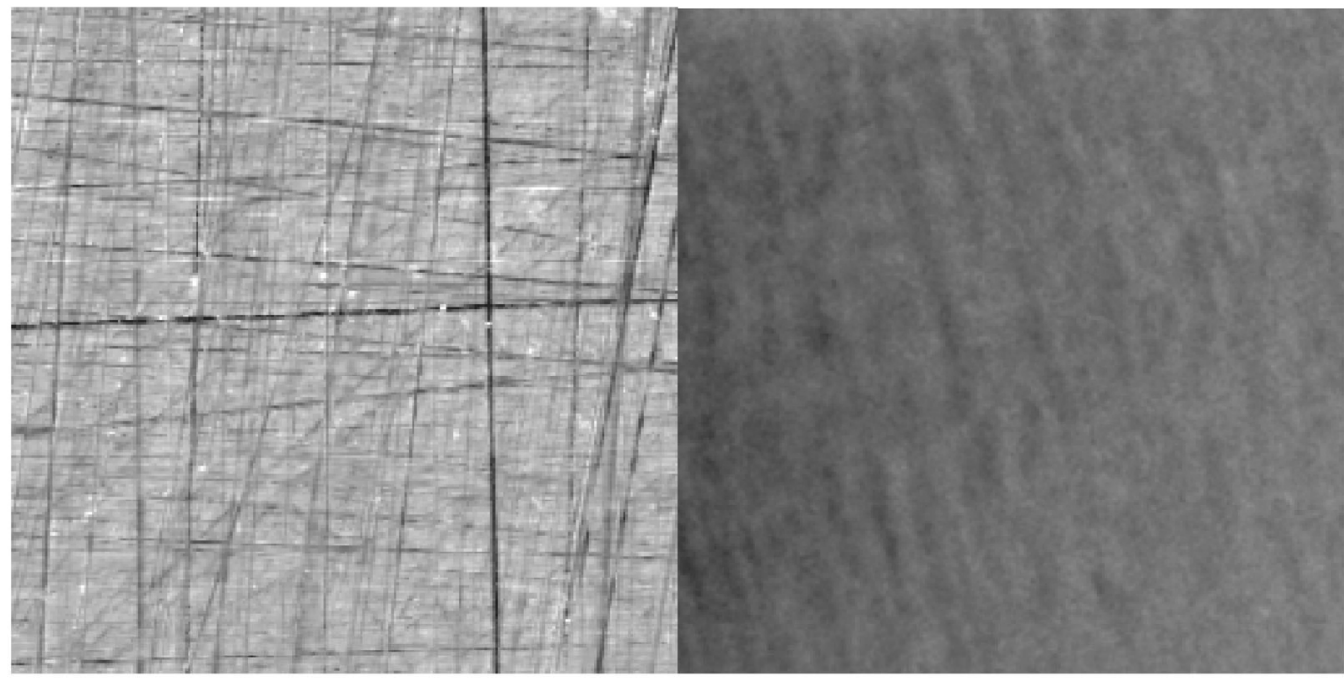

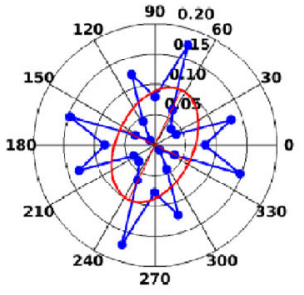

Scale no. 1

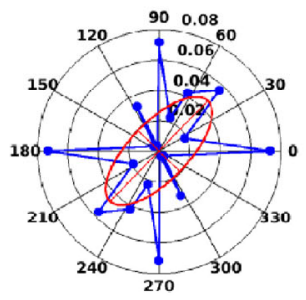

Scale no. 6

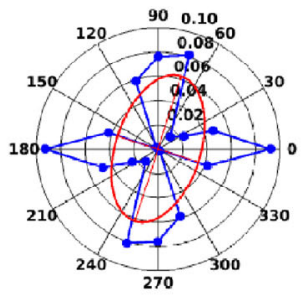

Scale no. 3

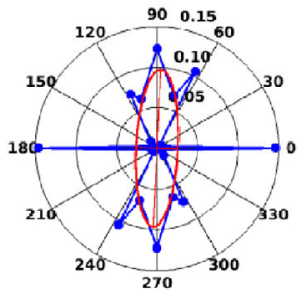

Scale no. 9

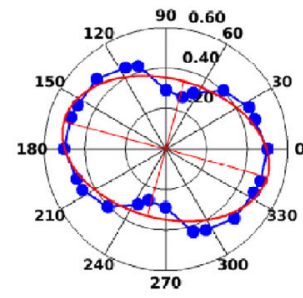

Scale no. 1

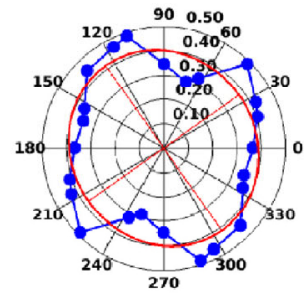

Scale no. 6

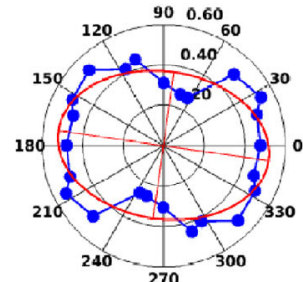

Scale no. 3

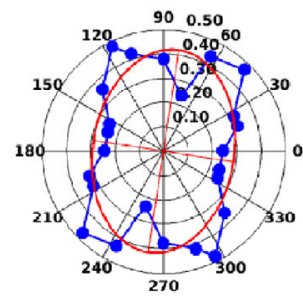

Scale no. 9

Fig. 6 Range image of the surface topography and x-ray region of interest of a normal knee together with the DFS analysis results.

Most of the tribological research has been focused on the replacements for hip and knee joints, as shown in Fig. 7. In 1881, Themistocles Glück used an ivory ball and a socket joint. Since then different materials, such as glass, steel, rubber, acrylic and materials combinations were used for hip joint replacements. It was quickly realized that the artificial joint material must not only be biocompatible, but also able to withstand the body stresses with minimum wear. The introduction of ultra-high-molecular-weight polyethylene as a bearing surface, by John Charnley in 1963, revolutionized the design of hip prostheses. It happened by pure chance. UHMWPE salesman thought that as the polyethylene was used to make gears, it should also work well in hip prostheses.
Charnley initially dismissed the UHMWPE, but his laboratory technician tested it and the results were good. This discovery has led to the development of modern hip and knee joint replacements.

Early designs of knee prostheses appeared in the 1940s as simple hinges. This basic design failed to accommodate the complexities of knee movement and the failure rate was very high. Rapid loosening and infection were major problems. The introduction of UHMWPE as a bearing surface and the provision for unrestricted rotational movement solved the early failure problem and resulted in a modern knee implant. Without the input from tribology the development of knee and hip implants, which provide pain relief and improved mobility to a large number of 

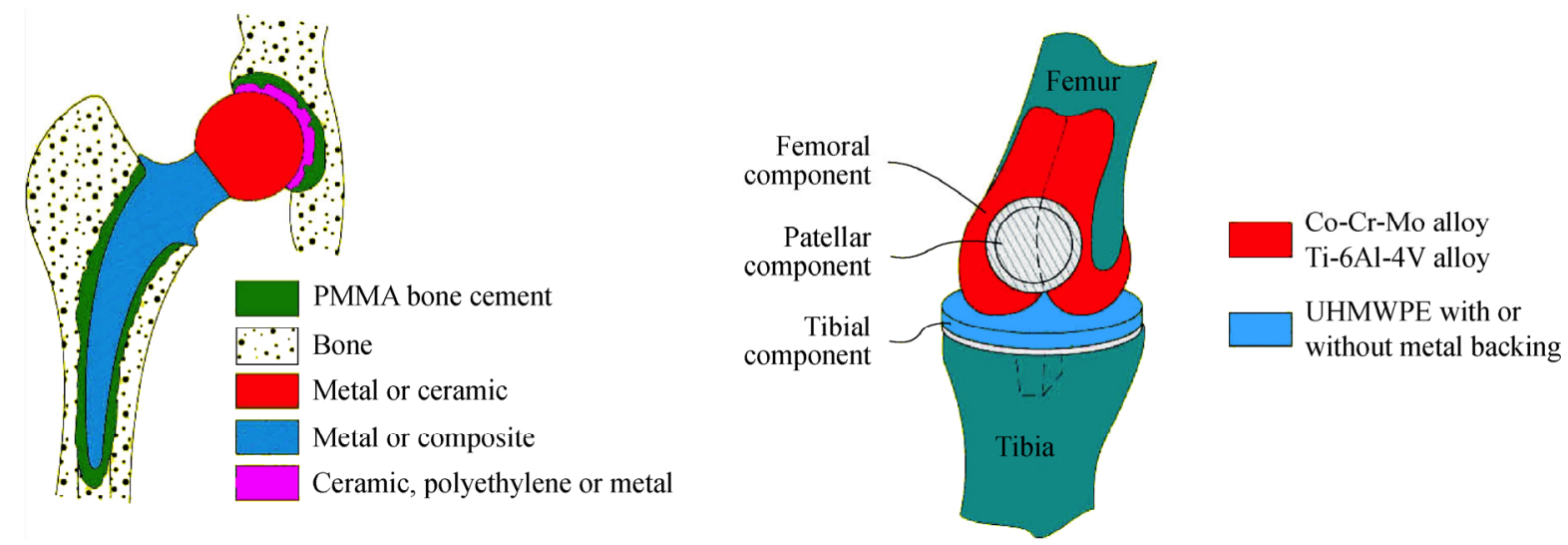

Fig. 7 Schematic diagrams of hip and knee implants (adapted from Ref. [52]).

osteoarthritis sufferers, would not be possible.

Tribology has also influenced the development of new dental restorative materials, which are fitted to an ever increasing number of patients, vascular prostheses such as artificial heart and others. Materials used for these devices notoriously suffer from wear problems.

\section{$5 \quad$ Environmental tribology}

Major global concerns now include rapid environmental degradation occurring across the globe, diminishing oil reserves and speedy climate change. As a response to these concerns environmental tribology has evolved with a research focus on innovative technologies, green lubricants and new materials aiming to reduce friction and wear with a bonus of reduction in energy consumption and the environmental impact.

The challenges that we face are not trivial. For example, a serious problem is the disposal of used mineral lubricating oils. These substances are often toxic and present in large volumes. To get a perspective, there are about 40 million tonnes of oils being discarded annually worldwide. Try to imagine a lake 4,000 $\mathrm{m}$ long, $500 \mathrm{~m}$ wide and $22 \mathrm{~m}$ deep [15]. Only a small proportion of the used oil is reprocessed and most of it is disposed back into the environment. Solution might be provided by biodegradable lubricating oils, which can be harmlessly decomposed by bacteria and fungi after use. Potential applications for biodegradable lubricants range from bulldozers and excavators which notoriously leak the hydraulic fluid during operation, to ships plagued by perennial problems of oil leakage to the sea.
Lubricants contain additives consisting of sulphur, phosphorus and many other, often toxic, compounds. Burning these additives with fuel in internal combustion engines, especially diesel, directly contributes to the acid rain and pollution in most of the cities across the globe. In some cities pollution is chronic and poses a severe health hazard. Apart from green lubricants material surfaces with artificially introduced textures are used to reduce friction and hence fuel consumption in engines.

\section{Nanotribology}

Device miniaturization is one of the frontier technologies of the 21st century. Introduction of micro and nanotechnologies may change the ways in which people and machines interact with the physical world. MEMS devices find applications in medicine, biotechnology, optics, electronics, aviation and many others. Mechanisms of material removal at nano/micro scale are becoming vital in the development of nano/ micro grinding technologies. These technologies are needed in the production of, for example, microlenses with good surface finish for miniaturized endoscopes or cell phones.

From nanotechnology, a research field of nanotribology has emerged, which is the study of tribology in minute contacts. This is a great leap from a macro to a nano scale tribology $[15,53]$. A major impact of nanotribology has been in the development of computer disk drives with high recording densities. The recording density is inversely proportional to the film thickness between the recording head and the memory 
disk. The film thickness between head and disk was initially provided by aerodynamic lubrication [15]. Monomolecular films, as thin as a few nanometers, instead of the conventional $0.1 \mu \mathrm{m}$ of air films [54], have been achieved, dramatically increasing the recording densities.

In nanotribology, it is no longer possible to apply continuum mechanics to the analysis of surface contacts; instead the contacting solids must be modelled as what they really are, a matrix of bonded atoms or ions. Atomistic (i.e., involving explicit models of atoms) molecular dynamics simulations have been used to model the sliding nano contact. Currently these studies are limited to a few nanometers of sliding distance and a few hundred of atoms, but with increased computer power such studies at a larger scale will become possible in the near future. Fundamental principles of tribology such as Amontons' law are now being analysed in detail by computational models, i.e., the forces and energy flows on each atom within a sliding contact are being computed. Initially, most of the work conducted on nanoscale has been related to friction but there is an increasing volume of studies on nanoscale wear [15]. There, the basic concepts of tribology would need to be revised. For example, how can there be wear particles when the size of the contact is much smaller than the average diameter of typical wear particles? Is plastic deformation possible in nano contacts when the contact diameter is less than the spacing between dislocations? In micro-machine, only zero or negligible wear is permissible or else the sliding components would seize [55].

As the reliable operation of minute contacts requires friction control, just like in larger contacts, nanolubrication is of importance. For nano contact, the traditional methods of lubrication are not suitable. New methods of lubrication, e.g., vapour phase, carbon molecules (bucky balls, nano-onions, etc.), non-volatile monomolecular films of lubricants (often self-assembled), are thus being developed [15].

\section{Summary}

Since its official introduction of the word "tribology" in the 1960s, the work of tribologists has primarily been focused on solving the immediate industrial problems of wear and friction through thorough understanding of the lubrication mechanisms and surface phenomena, and the application of better materials, novel surface technologies, improved lubricants and lubrication methods. Over the last fifty years, largely thanks to tribology, the fuel consumption in motor vehicles has almost halved with twofold power increase. This resulted in decreased pollution by an individual car unfortunately only to be counterbalanced by a dramatic increase of car users. Even though the beneficial effect was largely nullified, without tribology several cities across the world would likely reach now the pollution levels deemed unsafe for humans.

Advancements in tribology allowed for the construction of ships of monumental size, sending satellites and robots into space, development of modern data storage devices, revolution in the road and air transportation, etc. Like with any progress there is a price to pay as humanity is faced with the problems of greenhouse gas emissions, global warming and environment degradation.

Once the immediate industrial problems associated with lubrication, wear and friction have been solved, or at least attenuated, the research focus has shifted to the newly emerging areas like biotribology, environmental tribology and nanotribology. Biotribology has been developed as a result of the growing interest in applying scientific methods to understand tribological phenomena occurring in the living tissue. It seems that much of the future research will be shaped by our desire to prolong human life. As our bodies are not designed to last a hundred or more years, they would have to be augmented by the increasing numbers of components made from synthetic materials and tribology would be vital to their reliable operation.

Nanotribology is an integral part of nanotechnology. It involves studies of tribology within extremely small contacts. Atomistic molecular dynamics simulations are used to model the sliding contact to understand friction at nano scale. Environmental tribology has evolved as a response to global concerns related to the gradual environment degradation, diminishing oil reserves and rapid climate change. This has resulted in new research focused on innovative technologies, e.g., surface textures, green lubricants and advanced materials aiming to reduce friction and wear and, at 
the same time, reduce energy consumption and the environmental impact.

The increasing pace of research and development yields an urgent need to predict in advance what the future directions in tribology might be. For example, as affordable oil and gas resources are gradually diminishing the major issue is how to conserve what is still left and, at the same time how to provide increasing population with adequate mobility and sufficient power. Would we still be able to live in our cities?

As in the past, tribology research would help us to solve many of the issues arising and also it would help us to survive, e.g., by reducing energy consumption, managing pollution or contamination of the environment. Will there be major advances in our fundamental knowledge of tribology in the next fifty years? Will the range of tribological applications be significantly extended? Time will show. Over the last fifty years numerous scientists have advanced our tribological knowledge by establishing solid theoretical and practical foundations but it would be up to the younger generation to write future chapters in tribology. The issues in medical engineering, nano scale, longer machine lifetime, significant reduction in fuel consumption and improved energy conservation would need to be addressed very soon and this cannot be achieved without the tribology research.

Open Access: The articles published in this journal are distributed under the terms of the Creative Commons Attribution 4.0 International License (http:// creativecommons.org/licenses/by/4.0/), which permits unrestricted use, distribution, and reproduction in any medium, provided you give appropriate credit to the original author(s) and the source, provide a link to the Creative Commons license, and indicate if changes were made.

\section{References}

[1] Autumn K, Liang Y A, Hsieh S T, Zesch W, Chan W P, Kenny T W, Fearing R, Full R J. Adhesive force of a single gecko foot-hair. Nature 405: 681-685 (2000)

[2] Bhushan B. Adhesion of multi-level hierarchical attachment systems in gecko feet. Journal of Adhesion Science and Technology 21(12-13): 1213-1258 (2007)

[3] Cobb K. Getting a grip: How gecko toes stick. Science News
162: 133 (2002)

[4] Izadi H, Stewart K M E, Penlidis A. Role of contact electrification and electrostatic interactions in gecko adhesion. Journal of the Royal Society Interface 11(98): 20140371 (2014)

[5] Geim A K, Dubonos S V, Grigorieva I V, Novoselov K S, Zhukov A A, Shapoval Y S. Microfabricated adhesive mimicking gecko foot-hair. Nature Materials 2: 461-463 (2003)

[6] Buckley D H. Surface Effects in Adhesion, Friction, Wear and Lubrication. Amsterdam: Elsevier, 1970.

[7] Dowson D. History of Tribology, 2nd Edition. Professional Engineering Publishing, 1998.

[8] Tower B. First report on friction experiments. In Proc Inst Mech Eng, 1883: 632-669.

[9] Batchelor A W, Stachowiak G W. Revealing the hidden world of wear and friction. Mechanical Engineering Transactions, Journal of the Inst. of Engineers 20(1): 5-13 (1995)

[10] Reynolds O. On the theory of lubrication and its application to $\mathrm{Mr}$ Beauchamp Tower's experiments including an experimental determination of the viscosity of olive oil. Phil Trans Roy Soc London 177(Part I): 157-234 (1886)

[11] Ettles C M M, Akkok M, Cameron A. Inverse hydrodynamic methods applied to Mr Beauchamp Tower's experiments of 1885. Transactions ASME, Journal of Lubrication Technology 102: 172-181 (1980)

[12] Hertz H. Uber die beruhrung fester elastischer korper. J Reine und Angewandte Mathematik 92: 156-171 (1881)

[13] Martin H M. Lubrication of gear teeth. Engineering London 102: 199-221 (1916)

[14] Grubin A N. Fundamentals of the hydrodynamic theory of lubrication of heavily loaded cylindrical surfaces. in Investigation of the Contact Machine Components. Ketova K F ed. Translation of Russian Book No. 30, Central Scientific Institute for Technology and Mechanical Engineering, Moscow, 1949.

[15] Stachowiak G W, Batchelor A W. Engineering Tribology (Fourth Edition). Elsevier-Butterworth-Heinemann, 2013.

[16] Cameron A, Gohar R. Theoretical and experimental studies of the oil film in lubricated point contacts. Proc Roy Soc London Series A 291: 520-536 (1966)

[17] Cameron A, Gohar R. Optical measurement of oil film thickness under elastohydrodynamic lubrication. Nature 200: 458-459 (1963)

[18] Marx N, Guegan J, Spikes H A. Elastohydrodynamic film thickness of soft EHL contacts using optical interferometry. Tribology International 99: 267-277 (2016)

[19] Dowson D, Higginson C. Elastohydrodynamic Lubrication. Oxford: Pergamon Press, 1977.

[20] Johnston G J, Wayte R, Spikes H A. The measurement and study of very thin lubricant films in concentrated contacts. Tribol Trans 34(2): 187-194 (1991) 
[21] Cann P M, Spikes H A. The development of a spacer layer imaging method (SLIM) for mapping elastohydrodynamic contacts. Tribology Transactions 39: 915-921 (1996)

[22] Chua W-H, Stachowiak G W. The study of the dynamic thickness of organic boundary films under metallic sliding contact. Tribology Letters 39: 151-161 (2010)

[23] Woloszynski T, Podsiadlo P, Stachowiak G W. Efficient solution to the cavitation problem in hydrodynamic lubrication. Tribology Letters 58(1): 1-11 (2015)

[24] Hardy W B, Doubleday I. Boundary lubrication-The paraffin series. Proc Roy Soc London Series A 100: 550-574 (1921)

[25] Hardy W B, Doubleday I. Boundary lubrication-The temperature coefficient. Proc Roy Soc London Series A 101: 487-492 (1922)

[26] Bowden F P, Tabor D. The Friction and Lubrication of Solids, Part 1. Oxford: Clarendon Press, 1950.

[27] Hsu S M, Zhang J, Yin Z. The nature and origin of tribochemistry. Tribology Letters 13(2): 131-139 (2002)

[28] Hatchett C. Experiments and various observations on various alloys, on the specific gravity, and on the comparative wear of gold. Being the substance of a report made to the Right Honourable the Lord of the Committee of Privy Council, appointed to take into consideration the state of the coins of the Kingdom, and the present establishment and Constitution of his Majesty's Mint. Phil Trans R Soc London 93: 43-194 (1868)

[29] Desaguliers J T. A Course of Experimental Philosophy. London: John Senex, 1734.

[30] Bowden F P, Tabor D. The Friction and Lubrication of Solids. Oxford: Oxford University Press, 1964.

[31] Krim J. Friction at the atomic scale. Scientific American 275: 74-82 (1996)

[32] Leslie J. An Experimental Enquiry into the Nature and Propagation of Heat. Printed for J. Newman, No. 22, Poultry, T. Gillet Printer, Salisbury Square, United Kingdom, 1804.

[33] Stachowiak G W. Tribology research and enterprise in Australia. In World Tribology Congress, New Generation Tribology for Achieving a Sustainable Society. Japanese Society of Tribologists, 2005: 4-7.

[34] Challen J M, McLean L J, Oxley P L B. Plastic deformation in sliding contact with a hard wedge: Its relation to friction and wear. Proc Roy Soc London Series A 394: 161-181 (1984)

[35] Rigney D A, Hirth J P. Plastic deformation and sliding friction of metals. Wear 53: 345-370 (1979)

[36] Cocks M. Interaction of sliding metal surfaces. Journal of Applied Physics 33: 2152-2161 (1962)

[37] Antler M. Processes of metal transfer and wear. Wear 7: 181-204 (1964)

[38] Komvopoulos K, Saka N, Suh N P. The mechanism of friction in boundary lubrication. Transactions ASME, Journal of Tribology 107: 452-463 (1985)

[39] Knox S A, Harrison C M, Britt H C, Henderson J V. Estimating prevalence of common chronic morbidities in Australia. Med J Australia 189: 66-70 (2008)

[40] Access Economics. Painful realities: The economic impact of arthritis in Australia in 2007. Sydney: Arthritis Australia, 2007.

[41] Jones C, Stoffel K, Ozturk H, Stachowiak G W. The effects of surface active phospholipids on wear and lubrication of osteoarthritic sheep joints: Wear. Tribology Letters 16: 291-296 (2004)

[42] Ballantine G C, Stachowiak G W. The effects of lipid depletion on osteoarthritic wear. Wear 253: 385-393 (2002)

[43] Graindorge S L, Stachowiak G W. Changes occurring in the surface morphology of articular cartilage during wear. Wear 241: 143-151 (2000)

[44] Wolski M, Podsiadlo P, Stachowiak G W. Directional fractal signature analysis of trabecular bone evaluation of different methods to detect early osteoarthritis in knee radiographs. Journal of Engineering in Medicine, Proc Inst Mech Eng, Part H 223: 211-236 (2009)

[45] Wolski M, Podsiadlo P, Stachowiak G W. Applications of the variance orientation transform method to the multi-scale characterization of surface roughness and anisotropy. Tribology International 43: 2203-2215 (2010)

[46] Wolski M, Podsiadlo P, Stachowiak G W, Lohmander L S, Englund M. Differences in trabecular bone texture between knees with and without radiographic osteoarthritis detected by directional fractal signature method. Journal of Osteoarthritis and Cartilage 18: 684-690 (2010)

[47] Wolski M, Stachowiak G W, Dempsey A R, Mills P M, Cicuttini F M, Wang Y, Stoffel K K, llyod D G, Podsiadlo P. Trabecular bone texture detected by plain radiography and variance orientation transform method is different between knees with and without cartilage defects. Journal of Orthopaedic Research 29: 1161-1167 (2011)

[48] Wolski M, Podsiadlo P, Stachowiak G W. Directional fractal signature analysis of self-structured surface textures. Tribology Letters 47(3): 323-340 (2012)

[49] Woloszynski T, Podsiadlo P, Stachowiak G W, Kurzynski M, Lohmander L S, Englund M. Prediction of progression of radiographic knee osteoarthritis using tibial trabecular bone texture. Arthritis \& Rheumatism 64: 688-695 (2012)

[50] Wolski M, Podsiadlo P, Stachowiak G W. Analysis of AFM images of self-structured surface textures by directional fractal signature method. Tribology Letters 49: 465-480 (2013)

[51] Podsiadlo P, Stachowiak G W. Directional multiscale analysis and optimization for surface textures. Tribology Letters 49: 179-191 (2013) 
[52] Stachowiak G W. Friction and wear of polymers, ceramics and composites in biomedical applications. In Advances in Composites Tribology, Volume 8, Composite Materials Series. Friedrich K ed. Elsevier, 1993: 509-557.

[53] Bhushan B, Israelachvili J N, Landman U V. Nanotribology: Friction, wear and lubrication at the atomic scale. Nature 374: 607-616 (1995)

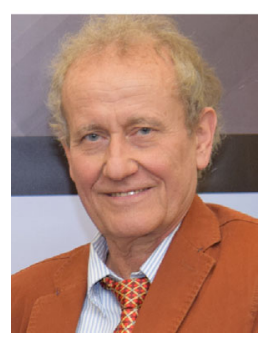

Gwidon STACHOWIAK. He is the Head of the Tribology Laboratory at the School of Mechanical and Civil Engineering, Curtin University in Western Australia. His current work is focused on the development of methods for the characterization of multiscale 3D surface topographies, prediction of osteoarthritis in knee joints based on X-ray images and tribocorrosion. He is the editor of Tribology and Practice book series published by John Wiley and member of the editorial boards of several tribological and bio-medical journals. He is also a member of several
[54] Karis T E. Nanotribology of thin film magnetic recording media. In Nanotribology, Critical Assessment and Research Needs. Hsu S M, Ying Z C ed. Dordrecht (The Netherlands): Kluwer Academic Publishers, 2003: 291-325.

[55] Hsu S M. Nanotribology: The link to macrotribology. In Proc. Intern. Tribology Conf., AUSTRIB '02, Perth, Australia, 2002: 9-15.

international committees including the Executive Committee of the International Energy Agency, Research and Development of Advanced Materials for Transportation. Professor Stachowiak has published extensively and wrote/contributed to several books. $\mathrm{He}$ is the leading author of the books "Engineering Tribology" and "Experimental Methods in Tribology" published by Elsevier. In 2014, he was awarded Tribology Gold Medal, the World's Highest Award in Tribology in recognition of his outstanding contribution to tribology while in 2012, he was awarded the title of Doctor Honoris Causa from the Ecole Centrale de Lyon, France. 